\title{
Quantum bounce and cosmic recall
}

\author{
Alejandro Corichi ${ }^{\dagger, \star}$ and Parampreet Singh ${ }^{\ddagger}, \star \star$ \\ ${ }^{\dagger}$ Instituto de Matemáticas, Unidad Morelia, Universidad Nacional Autónoma de México, \\ UNAM-Campus Morelia, A. Postal 61-3, Morelia, Michoacán 58090, Mexico \\ ${ }^{\star}$ Center for Fundamental Theory, Institute for Gravitation and the Cosmos, Penn State, University Park PA 16802, USA \\ $\ddagger$ Perimeter Institute for Theoretical Physics, 31 Caroline Street North, Waterloo, Ontario N2L 2Y5, Canada
}

\begin{abstract}
Loop quantum cosmology predicts that, in simple models, the big bang singularity of classical general relativity is replaced by a quantum bounce. Because of the extreme physical conditions near the bounce, a natural question is whether the universe can retain, after the bounce, its memory about the previous epoch. More precisely, does the universe retain various properties of the state after evolving unitarily through the bounce or does it suffer from some cosmic amnesia as has been recently suggested? Here we show that this issue can be answered unambiguously at least within an exactly solvable model, derived from a small simplification of loop quantum cosmology, for which there is full analytical control on the quantum theory. We show that if there exists a semiclassical state at late times on one side, peaked around a pair of canonically conjugate variables, then there are very strong bounds on the fluctuations on the other side of the bounce, implying semi-classicality. For such a model universe which grows to a size of 1 megaparsec,the change in relative fluctuations of the only non-trivial observable of the model across the bounce is less than $10^{-56}$ (becoming smaller for universes which grow larger). The universe maintains (an almost) total recall.
\end{abstract}

The singularity theorems of classical general relativity (GR) have taught us that the homogeneous cosmological solutions possessing a singularity are examples of a generic feature of the theory, rather than an artifact of the symmetries they exhibit [1]. In quantum gravity we do not yet have a general result on the fate of the classical singularities. The detailed study of isotropic solutions in a symmetry reduced quantum theory of gravity known as loop quantum cosmology (LQC) [2] has shown that for a universe filled with a massless scalar field, with or without a cosmological constant, the singularity gets replaced by a quantum bounce [3]. Whether singularity resolution is an artifact of the symmetries or a generic feature of quantum cosmology is an open question. However, homogeneous models are excellent approximation to our Universe at large scales and recent analytical and numerical studies in GR strongly suggest that homogeneous modes dictate the dynamics near space-like singularities (thus confirming the Belinskii-Khalatnikov-Lifshitz conjecture). Investigations of simple models as above, even with their intrinsic limitations, thus provide valuable insights on difficult questions such as the nature of spacetime across classical singularities. Just as in other instances in physics, one could expect that qualitative features of these models survive in more general situations. Preliminary studies of anisotropic models within LQC suggest that such expectations are justified [4].

Within this model, extensive analytical and numerical investigations have revealed various features of the quantum spacetime across the bounce [3, [5, [6]. Due to the complicated form of the quantum constraint, full analytical control of LQC is difficult, so it is natural to look for slightly simpler models that capture the physical content of the theory but that are amenable for analytical investigations. Recently, such a model based on a small simplification of the quantum constraint in $k=0$ isotropic LQC has been introduced [7]. It is exactly solvable and replicates all the features of LQC for universes which grow to a large size to a great accuracy. This model describes homogeneous and isotropic flat universe sourced with a massless scalar field $\phi$ (which plays the role of internal clock). The exactly solvable model of LQC (sLQC) not only confirms the result of the quantum bounce for semi-classical states as first seen in the numerical simulations [3] but extends them to almost all the states of the physical Hilbert space. Further, the quantum constraint approximates the classical one at both early and late times. Various questions which could not be fully answered in LQC, can now be posed in sLQC.

Since the curvatures are so large at the bounce, it is natural to inquire whether significant 'information' can be lost across it. Such a possibility was recently highlighted in [8] where it was argued that a universe such as ours, which is semi-classical to an excellent degree of approximation, could have nonetheless evolved from a state which has an arbitrarily large (and unconstrained) relative dispersions at early times much before the bounce. If this were possible, by making observations today or in the future we would never be able to determine the initial, pre-bounce state of the universe. The purpose of this Letter is to address this question within sLQC.

Our analysis is based on this simplified model that features, however, two significant improvements over that of $[8,9,10]$. First, it is very closely related to LQC through a simple, well motivated and well controlled approximation of the quantum constraint. Secondly, sLQC agrees with general relativity at low curvatures and is free from gauge artifacts. Further, our analysis is not tied to the coherence properties of the state which is a stronger assumption than semi-classicality which only re- 
quires for an observable $\mathcal{O}$ that its expectation value be very close to the classical one and the relative fluctuation $(\Delta \hat{\mathcal{O}})^{2} /\langle\hat{\mathcal{O}}\rangle^{2} \ll 1$. We will show that the relative fluctuations in the initial semi-classical state peaked in conjugate variables, strongly bound the relative fluctuations on the other side of the bounce. More precisely the relative fluctuation of the Dirac observable $\left.\hat{V}\right|_{\phi}$ (volume at a given instant of 'time' $\phi=\phi_{o}$ ) across the bounce satisfies the above inequality if the initial state was peaked in a large classical universe. In fact, for a large class of states the change in relative dispersion is zero. Since the field $\phi$ is massless, the expectation value of its momentum $\hat{p}_{\phi}$ and corresponding fluctuations characterizing the state are trivially conserved across the bounce. Thus, above results show that a state which is semi-classical at late (early) times, preserves semi-classicality at early (late) times across the bounce.

Let us recall the model we are considering. In terms of the phase space variables used in loop quantum gravity (LQG) [11], namely a connection $A_{a}^{i}$ and a densitized triad $E_{i}^{a}$, the homogeneous sector can be expressed as,

$$
A_{a}^{i}=c V_{0}^{-(1 / 3) \mathrm{o}} \omega_{a}^{i} \quad ; \quad E_{i}^{a}=p V_{0}^{-(2 / 3)} \sqrt{q_{0}} \mathrm{o} e_{i}^{a}
$$

where $\left({ }^{\mathrm{o}} \omega_{a}^{i},{ }^{\mathrm{o}} e_{i}^{a}\right)$ are a set of orthonormal co-triads and triads compatible with the fiducial (flat for $k=0$ ) metric ${ }^{\circ} q_{a b}$ and $V_{o}$ is the volume of the fiducial cell, introduced to define sympectic structure, with respect to ${ }^{\circ} q_{a b}$. The phase space is characterized by conjugate variables $(c, p)$ satisfying $\{c, p\}=(8 \pi G \gamma) / 3$. where $\gamma$ is the BarberoImmirzi parameter. Triad $p$ is related to the physical volume $V$ as $|p|=V^{2 / 3}=V_{o}^{2 / 3} a^{2}$ where $a$ is the scale factor and on the space of classical solutions $c=\gamma \dot{a}$. It is convenient to introduce [7]

$$
\mathrm{b}:=\varepsilon c / p^{1 / 2} \quad \text { and } \quad \nu=\varepsilon p^{3 / 2} /\left(2 \pi \ell_{\mathrm{Pl}}^{2} \gamma\right)
$$

where $\hbar\{\mathrm{b}, \nu\}=2$. Here $\varepsilon= \pm 1$ is the orientation of the triad with respect to that of ${ }^{\circ} \omega_{a}^{i}$. The classical constraint then becomes $\mathrm{b}^{2} V^{2} / \gamma^{2}=(8 \pi G / 3) p_{\phi}^{2} / 2$. In the $\mathrm{b}$ representation, the quantum constraint of sLQC becomes [7],

$$
\frac{\partial^{2}}{\partial \phi^{2}} \cdot \chi(\mathrm{b}, \phi)=\beta^{2}\left(\frac{\sin (\lambda \mathrm{b})}{\lambda} \frac{\partial}{\partial \mathrm{b}}\right)^{2} \cdot \chi(\mathrm{b}, \phi)
$$

with $\mathrm{b} \in(0, \pi / \lambda), \beta:=\sqrt{12 \pi G}$ and $\lambda^{2}=2 \sqrt{3} \pi \gamma \ell_{\mathrm{Pl}}^{2}[7]$. By introducing

$$
x=\beta^{-1} \ln (\tan (\lambda \mathrm{b} / 2))
$$

the quantum constraint can be rewritten in a KleinGordon form

$$
\partial_{\phi}^{2} \chi(x, \phi)=\partial_{x}^{2} \chi(x, \phi)
$$

A general solution $\chi(x, \phi)$ to the above equation can be decomposed in the left and right moving components:

$$
\chi=\chi_{+}(\phi+x)+\chi_{-}(\phi-x):=\chi_{+}\left(x_{+}\right)+\chi_{-}\left(x_{-}\right) .
$$

The physical states are positive frequency solutions of (6). Since there are no fermions in the model, the orientations of the triad are indistinguishable and $\chi(x, \phi)$ satisfy the symmetry requirement $\chi(-x, \phi)=-\chi(-x, \phi)$. Thus, $\chi(x, \phi)=\left(F\left(x_{+}\right)-F\left(x_{-}\right)\right) / \sqrt{2}$, where $F$ is an arbitrary positive frequency solution. The physical inner product given as [7]

$$
\left(\chi_{1}, \chi_{2}\right)_{\mathrm{phy}}=-2 i \int_{-\infty}^{\infty} \mathrm{d} x \bar{F}(x) \partial_{x} F(x) .
$$

We can now compute the expectation values and fluctuations of $\left.\hat{V}\right|_{\phi_{o}}$. For any state of the physical Hilbert space

$$
\begin{aligned}
\langle\hat{V}\rangle_{\phi} & =V_{+} e^{\beta \phi}+V_{-} e^{-\beta \phi} \\
\left\langle\hat{V}^{2}\right\rangle_{\phi} & =W_{0}+W_{+} e^{2 \beta \phi}+W_{-} e^{-2 \beta \phi} \\
(\Delta \hat{V})_{\phi}^{2} & =Y_{0}+Y_{+} e^{2 \beta \phi}+Y_{-} e^{-2 \beta \phi}
\end{aligned}
$$

with $V_{ \pm}, W_{0}, W_{ \pm}, Y_{0}$ and $Y_{ \pm}$being real and positive, given by

$$
\begin{gathered}
V_{ \pm}=\frac{4 \pi \gamma \ell_{\mathrm{Pl}}^{2} \lambda}{\beta} \int \mathrm{d} x\left|\frac{\mathrm{d} F}{\mathrm{~d} x}\right|^{2} e^{\mp \beta x} \\
W_{0}=\frac{i \pi \gamma^{2} \ell_{\mathrm{Pl}}^{4} \lambda^{2}}{3 G} \int \mathrm{d} x\left(\frac{\mathrm{d}^{2} \bar{F}}{\mathrm{~d} x^{2}} \frac{\mathrm{d} F}{\mathrm{~d} x}-\frac{\mathrm{d} \bar{F}}{\mathrm{~d} x} \frac{\mathrm{d}^{2} F}{\mathrm{~d} x^{2}}\right) \\
W_{ \pm}=\frac{i \pi \gamma^{2} \ell_{\mathrm{Pl}}^{4} \lambda^{2}}{6 G} \int \mathrm{d} x e^{\mp 2 \beta x}\left(\frac{\mathrm{d}^{2} \bar{F}}{\mathrm{~d} x^{2}} \frac{\mathrm{d} F}{\mathrm{~d} x}-\frac{\mathrm{d} \bar{F}}{\mathrm{~d} x} \frac{\mathrm{d}^{2} F}{\mathrm{~d} x^{2}}\right)
\end{gathered}
$$

$Y_{0}=W_{0}-2 V_{+} V_{-}$and $Y_{ \pm}=W_{ \pm}-V_{ \pm}^{2}$.

From (8), it follows that the expectation value of $\left.\hat{V}\right|_{\phi}$ is large at both very early and late times and has a non-zero global minimum $V_{\min }=2\left(V_{+} V_{-}\right)^{1 / 2} /\|\chi\|^{2}$ occurring at bounce time $\phi_{b}^{V}=(2 \beta)^{-1} \ln \left(V_{-} / V_{+}\right)[7,[9]$. Around $\phi=$ $\phi_{b}^{V},\langle\hat{V}\rangle_{\phi}$ is symmetric. Similarly, $\left\langle\hat{V}^{2}\right\rangle_{\phi}$ is symmetric across $\phi_{b}^{V^{2}}=(4 \beta)^{-1} \ln \left(W_{-} / W_{+}\right)$. The first resulting observation is that if $\phi_{b}^{V}=\phi_{b}^{V^{2}}$, the difference in the asymptotic values of the relative fluctuation

$$
D:=\lim _{\phi \rightarrow \infty}\left[\left(\frac{(\Delta \hat{V})}{\langle\hat{V}\rangle}\right)_{-\phi}^{2}-\left(\frac{(\Delta \hat{V})}{\langle\hat{V}\rangle}\right)_{\phi}^{2}\right]=\frac{W_{-}}{V_{-}^{2}}-\frac{W_{+}}{V_{+}^{2}}
$$

vanishes.

We now show that for a large class of states, the difference between the relative fluctuations $D$ is indeed zero. We first express a physical state in terms of $F$

$$
\chi(x, \phi)=2 i \int \mathrm{d} k \tilde{F}(k) e^{-i k \phi} \sin (k x) .
$$

In the $\nu$ representation: $\tilde{e}_{k}(\nu)=\nu \mathcal{F}^{-1}\left[e_{k}(x(b))\right]$, with $e_{k}(x)=\sin (k x)$ and where $\mathcal{F}$ denotes the Fourier transform. Note that $\tilde{e}_{k}(\nu)$ is imaginary and symmetric in $\nu$. 
Hence, if $\tilde{F}(k)$ is real, then $\Psi(\nu, \phi)=\overline{\Psi(\nu,-\phi)}$. We can now evaluate the fluctuations:

$$
\begin{aligned}
(\Delta \hat{V})^{2}(\phi) & =\left\langle\Psi\left|(\hat{V}-\bar{V})^{2}\right| \Psi\right\rangle_{\text {phy }} \\
& =\sum_{m}\left|V_{m}-\bar{V}\right|^{2} \overline{\Psi\left(\nu_{m}, \phi\right)} \Psi\left(\nu_{m}, \phi\right)
\end{aligned}
$$

which show that if $\tilde{F}(k)$ is real, then $(\Delta \hat{V})_{\phi}^{2}=(\Delta \hat{V})_{-\phi}^{2}$. In fact, it turns out that the relative fluctuations are also symmetric (given that, by the same argument, $\langle\hat{V}\rangle(\phi)=$ $\langle\hat{V}\rangle(-\phi))$. Further, it is straightforward to see that this argument also extends to the initial state (in $k$ ) $\tilde{F}(k) e^{i k x_{0}}$, which in ' $x$ representation' is 'peaked' around $x=x_{0}$ (or is displaced). In this case, the fluctuations are symmetric with respect to the time $\left(\phi-x_{0}\right)$. Note that, this class of symmetric states, includes any real linear combination $\sum_{n} \alpha_{n} f_{n}(k)$ of functions of the form $f_{n}(k)=k^{n} e^{-\left(k-k_{0}\right)^{2} / \beta^{2}+i k x_{0}}$. As examples, squeezed states with arbitrary squeezing, and states having a Poisson distribution belong to this class. All such states preserve the relative fluctuation across the bounce. (A result that holds for dynamical coherent states 9,10$]$ ).

It is however possible that a realistic model universe is described by a state which does not belong to the above class and for which $D$ would not vanish. We now consider such states describing at late times a low curvature, large classical universe. For concreteness we wish to look at the evolution of fluctuations as we look backwards through the bounce from the expanding branch. A state describing the present (and future) late time epoch shall be sharply peaked on the classical trajectories and thus the relative dispersion in $\left.\hat{V}\right|_{\phi}$ shall satisfy:

$$
\lim _{\phi \rightarrow \infty}\left(\Delta \hat{V}_{\phi} /\langle\hat{V}\rangle_{\phi}\right)^{2}=W_{+} / V_{+}^{2}-1=: C-1=: \delta \ll 1 .
$$

that has to be compared with the asymptotic value of the relative dispersion 'before the big bang':

$$
\lim _{\phi \rightarrow-\infty}\left(\Delta \hat{V}_{\phi} /\langle\hat{V}\rangle_{\phi}\right)^{2}=W_{-} / V_{-}^{2}-1=: B-1 .
$$

The quantity of interest is $D=B-C$, introduced in (12). To evaluate it, let us consider the ratio of $B$ and $C, E:=B / C=\left(W_{-} V_{+}^{2}\right) /\left(W_{+} V_{-}^{2}\right)$. Let us assume that the relative fluctuations are larger on the other side of the bounce (the pre-big branch in the considered case). Then $E$ is greater than unity and we parameterize it as $E:=(1+\tilde{\Delta})$. We now show that if the dispersion of canonically conjugate variable to $\hat{V}$ and the relative dispersion of $\hat{V}$ in the present epoch is small $(\delta \ll 1)$, then both of the quantities $\tilde{\Delta}$ and $D$ are small. This in turn will imply a full control on the fluctuations of the universe 'before the big bang'.

Let us start by considering, as initial data, wave functions in $x$ that have most of its support in the interval $\left(x_{0}-\epsilon, x_{0}+\epsilon\right)$, around the point $x_{0}$. Intuitively, the quantity $\epsilon$ is proportional to spread or the absolute dispersion $\Delta x$ and using (4) to the relative dispersion $\Delta \tan (\lambda \mathrm{b} / 2) / \tan (\lambda \mathrm{b} / 2)$. At low curvature scales, $\mathrm{b} \ll 2 / \lambda$, and thus $\epsilon$ becomes proportional to $\Delta \mathrm{b} / \mathrm{b}$, i.e. the relative dispersion in the conjugate variable to $V$. If the state has to describe a universe at low curvature scales, $\epsilon$ is required to be small.

In order to put bounds on $\tilde{\Delta}$ we note that there exists some freedom in the choice of initial state. This freedom is in the choice of time $\phi$ at which the quantities are computed. After all, the wave-functions propagate along characteristics $x= \pm \phi$ preserving its shape (a feature responsible for invariance of $\left\langle\hat{p}_{\phi}\right\rangle$ and $\left.\left(\Delta \hat{p}_{\phi}\right)^{2}\right)$. We use this freedom, and without loss of generality, go to the time $\phi=\phi_{b}^{V^{2}}$, that is, the time at which $\left\langle\hat{V}^{2}\right\rangle_{\phi}$ bounces, where the $V$ 's and $W$ 's are computed. With this choice $W_{+}=W_{-}$.

With this choice, the problem is simplified and we only have to consider the ratio $1+\tilde{\Delta}=V_{+}^{2} / V_{-}^{2}$. Using (11) for the constants $V_{ \pm}$, we can then put bounds when the state is supported in the interval $\left(x_{0}-\epsilon, x_{0}+\epsilon\right)$ :

$$
V_{+}<N e^{-\beta\left(x_{0}-\epsilon\right)} \quad ; \quad V_{-}>N e^{\beta\left(x_{0}-\epsilon\right)}
$$

with $N=\frac{16 \gamma \ell_{\mathrm{P}}^{2} \lambda^{2}}{\beta} \int \mathrm{d} x\left|\frac{\mathrm{d} F}{\mathrm{~d} x}\right|^{2}$. We thus obtain,

$$
1+\tilde{\Delta}=\frac{V_{+}^{2}}{V_{-}^{2}}<e^{-4 \beta\left(x_{0}-\epsilon\right)} .
$$

Unless $x_{0}$ is bounded, this inequality provides little insights. For $W_{+}=W_{-}$, consistency of equations demands that $0 \in\left(x_{0}-\epsilon, x_{0}+\epsilon\right)$. From (11) it is easy to see that if this were not the case, we would have the same function weighted with functions that are larger than one for, say, $W_{+}$and smaller than one for $W_{-}$. The integrals would thus be different, contradicting $W_{+}=W_{-}$. Hence, $0 \in\left(x_{0}-\epsilon, x_{0}+\epsilon\right)$. Thus, it follows that $\epsilon>\left|x_{0}\right|$, from which we have, $1+\tilde{\Delta}=\frac{V_{+}^{2}}{V_{-}^{2}}<e^{8 \beta \epsilon}$ and therefore, $\tilde{\Delta}<e^{8 \beta \epsilon}-1$. Finally, we obtain

$$
\begin{aligned}
D & =B-C=(1+\tilde{\Delta}) C-C=\tilde{\Delta} C \\
& =(1+\delta) \tilde{\Delta}<(1+\delta)\left(e^{8 \beta \epsilon}-1\right)
\end{aligned}
$$

which is arbitrary small when both $\delta$ and $\epsilon$ are small. Since, $\delta$ and $\epsilon$ are measures of relative dispersions in volume $V$ and curvature $\mathrm{b}$ respectively, a state describing a large low curvature universe indeed have $\delta$ and $\epsilon$ very small. Thus, a state which is sharply in $\left.V\right|_{\phi}$ and its conjugate variable (or in $p_{\phi}$ for solutions to the constraint) on one side of the bounce is also sharply peaked on the other side.

We are now in position to answer the primary question of this Letter. Does this model universe retain its semiclassicality across the bounce? What we have shown is that for a state that is semiclassical at late times and for which the fluctuations in the volume are not symmetric across the bounce, there is nevertheless a strong 
bound on the possible dispersion on the 'other side of the bounce', making again the state semiclassical 'at early times'. This result was obtained within a simple but completely solvable model (sLQC) for which there is full control on the quantities at play. Note that our bounds are stringent only for states which are truly semi-classical at late times; they do not tell us much about states which are sharply peaked in volume but not its conjugate variable.

For the states of interest which describe at late times a large, low curvature universe both $\beta \epsilon$ and $\delta$ are small and to the leading order the inequality (18) yields $D<8 \beta \epsilon$. The value of $\epsilon$ in a classical universe can be heuristically estimated as follows. We first note that for sharply peaked states using (4), it follows that $\beta \epsilon \sim$ $\beta \Delta x \sim(\Delta \tan (\lambda \mathrm{b} / 2)) / \tan (\lambda \mathrm{b} / 2)$. At low curvatures, $\tan (\lambda \mathrm{b} / 2) \approx \lambda \mathrm{b} / 2$ and hence $\beta \epsilon \sim(\Delta \mathrm{b}) / \mathrm{b}$. Introducing a parameter $\alpha_{1}$ which measures the departure from minimum uncertainty between $\mathrm{b}$ and $\nu$, such that $\Delta \mathrm{b} \Delta \nu \approx 2 \alpha_{1}$, for the state peaked on the above classical trajectory

$$
(\Delta \mathrm{b} / \mathrm{b})(\Delta \nu / \nu) \approx \sqrt{12 \pi / G} \alpha_{1} \ell_{\mathrm{Pl}}^{2} / p_{\phi}
$$

which leads to (in classical $c=G=1$ units)

$$
(\Delta \mathrm{b} / \mathrm{b})^{2} \approx \sqrt{12 \pi} \alpha_{1} \alpha_{2}\left(\hbar / p_{\phi}\right)
$$

where $\alpha_{2}$ is a parameter which measures the ratio of relative dispersions in the conjugate variables: $\alpha_{2}:=$ $(\Delta \mathrm{b} / \mathrm{b}) /(\Delta \nu / \nu)$.

In our units, $p_{\phi}$ has dimensions of $\hbar$, thus for a universe to have a classical interpretation: $p_{\phi} \gg \hbar$ (implying $\Delta \mathrm{b} / \mathrm{b} \ll 1)$. In order to estimate $p_{\phi}$ it is useful to consider a closed universe which grows to a maximum size of 1 megaparsec. Using Friedman equation, the value of $p_{\phi}$ for the scalar field filling such a universe is $p_{\phi} \sim 1.8 \times 10^{114} \hbar$. Thus, for the initial state peaked at a classical trajectory in a 1 megaparsec universe: $\beta \epsilon \sim(\Delta \mathrm{b} / \mathrm{b}) \approx 2 \sqrt{\alpha_{1} \alpha_{2}} \times 10^{-57}$. Taking $\left(\alpha_{1}, \alpha_{2}\right) \sim O(1)$ for the initial semi-classical state, we obtain $D<10^{-56}$, which is an extremely small number. Thus across the bounce, $\left(\Delta \hat{V}_{\phi} /\langle\hat{V}\rangle_{\phi}\right)^{2} \ll 1$ and the state retains semi-classicality. A similar calculation for a universe with a size resembling ours, yields the change in relative fluctuations $D<10^{-60}$. Hence, within this model, the variation in the relative dispersion for a 'realistic universe' across the bounce is negligible. The universe recalls the properties of the semi-classical nature of the initial state to a large degree.

We note that above estimates only provide an upper bound on the change in relative fluctuation for the states which satisfy generic semi-classicality requirements initially. They are consistent with the numerical simulations with more specific states [3, 5]. Also, one should be careful in interpreting the results. From the above, one may be tempted to conclude that since absolute fluctuations may change significantly, there may be a loss of semi-classicality across the bounce. That such a conclusion is not realized becomes clear once one computes relative fluctuations of the observables which encode peakedness properties of the state.

To summarize, we have shown that, within a simplified and completely solvable model of LQC, if the universe is semiclassical at late times, that is, if it has very small relative dispersions in both relevant canonically conjugate variables, then the universe 'before the big bang' at early times is also semiclassical. To be precise, there is a strong bound on the possible relative dispersion 'on the other side' when the state is known to have, at late times, small relative dispersion on canonically conjugate variables. This result contributes significantly to the robustness of the SLQC scenario regarding singularity resolution [7], and shows that the possibilities highlighted in [8] are not realized. A question which remains open is the variation of higher moments quantifying more detailed properties of the state. Lessons learnt from the behavior of fluctuations give us valuable insights on their expected behavior, in particular that for realistic universes such variations may be bounded by very small numbers.

We thank A. Ashtekar for extensive discussions and helpful comments and T. Pawlowski for various discussions. This work was in part supported by CONACyT U47857F, NSF PHY04-56913 grants and by the Eberly Research Funds of Penn State. Research at Perimeter Institute is supported by the Government of Canada through Industry Canada and by the Province of Ontario through the Ministry of Research \& Innovation.

[1] S.W. Hawking and G.F.R. Ellis, The Large Scale Structure of Space-Time, (Cambridge, 1975).

[2] A. Ashtekar, Nuovo Cimento 112B, 1 (2007). M. Bojowald, Living Rev. Rel. 8, 11 (2005); A. Ashtekar, M. Bojowald and L. Lewandowski, Adv. Theor. Math. Phys. 7233 (2003)

[3] A. Ashtekar, T. Pawlowski and P. Singh, Phys. Rev. Lett. 96, 141301 (2006); Phys. Rev. D 74, 084003 (2006)

[4] D-W. Chiou, Phys. Rev. D 75, 024029 (2007).

[5] A. Ashtekar, T. Pawlowski, P. Singh, K. Vandersloot, Phys. Rev. D 75, 024035 (2007).

[6] W. Kaminski and J. Lewandowski, Class. Quant. Grav. 25, 035001 (2008).

[7] A. Ashtekar, A. Corichi, P. Singh, Phys. Rev. D 77, 024046 (2008).

[8] M. Bojowald, Nature Physics 3, 523 - 525 (01 Aug 2007).

[9] M. Bojowald, Phys. Rev. D 74, 081301 (2007); Phys. Rev. D 75, 123512 (2007).

[10] M. Bojowald, "Harmonic Cosmology: How much can we know about a universe before the big bang?", arXiv:0710.4919v1 [gr-qc].

[11] A. Ashtekar, J. Lewandowski, Class. Quant. Grav. 21, R53 (2004); C. Rovelli, Quantum Gravity, (Cambridge, 2004); T. Thiemann, Modern Canonical Quantum General Relativity, (Cambridge, 2007). 